\title{
Synthesis and Anticonvulsant Evaluation of 5-Vinyl-pyrrolidin-2-ones: A Potential GABA-AT Inhibitor
}

\author{
Tae-Woo Kwon, ${ }^{\star}$ Pan-Suk Kwon, Suk-Jin Song, Min-Soo Park, ${ }^{\dagger}$ and Jong-Won Choi ${ }^{\dagger}$ \\ Department of Chemistry and College of Phamacy, Kyungstong University, \\ 110-1, Daeyeon-dong, Nam-ku, Busan 608-736, Korea \\ Received Febriary: 23, 2002
}

Keywords : GABA-AT, Vigabatrin, 5-Vinyl-2-pyrrolidin-2-ones, Anticonvulsant activity.

4-Aminobutyric acid (GABA), 1, is well known for an important neurotransmitter in mammalian central nervous system (CNS). ${ }^{1}$ GABA deficiency has been associated with a variety of neurological disorders including Parkinson's disease, ${ }^{2}$ epilepsy, ${ }^{3}$ Schizophrenia, ${ }^{3}$ Huntington's chorea ${ }^{4}$ and tardive dyskinesia." Since GABA itself does not effectively penetrate the blood-brain-barrier, there has been a need for pro-drug of GABA or GABA-aminotransferase (GABA-AT) inhibitors that could enhance the concentration of GABA in brain. ${ }^{6}$ GABA-AT is a pyridoxal phosphate (PyCHO) dependent enzyme and vigabatrin, 2, is a typical GABA-AT inhibitor in clinical use for the treatment of epilepsy. The mechanism for the inhibition of aminobutyrate transaminase by vigabatrin, 2 , was proposed previously in which schiff base, 3, formed from vigabatrin and $\mathrm{PyCHO}$ and then a Michael type addition of nucleophiles in the enzyme followed as shown in $4^{7}$ On the basis of the mechanism mentioned, we envisioned that $N$-alkenyl lactam will increase lipophilicity to cross the blood-brain-barrier and act as a substrate for the enzyme enhanced nucleophilic addition by opening of ring via deprotecting sequence of $N$ alkenyl group. ${ }^{8}$<smiles>[R]C(N)CCC(=O)O</smiles>

$1 \cdot \mathrm{R}=\mathrm{II}$

2. $\mathrm{R}=\mathrm{CH}-\mathrm{CH}_{2}$

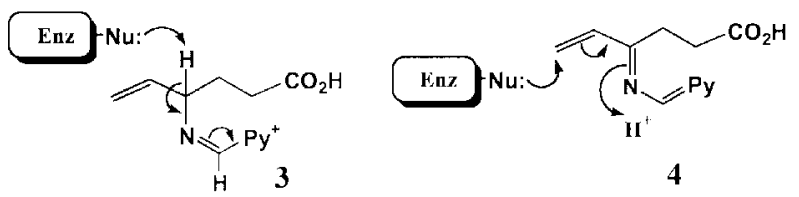

In conjunction with development of GABA related prodrug, a series of work on the synthesis of 5-vinyl-pyrrolidin2-ones" have been carried in our laboratory to investigate if the anticonvulsant activity appears to be influenced by variation of the $N$-alkenyl group. Here we report our results concerning synthesis and anticonvulsant evaluation of 5 vinyl-2-pyrrolidinone analogues, 17,18 and 19 prepared from L-glutamic acid.

The route to $N$-alkenyl pyrrolidin-2-one derivatives, 17 19, is shown in Scheme 1. Lactam, 7, was obtained in $98 \%$

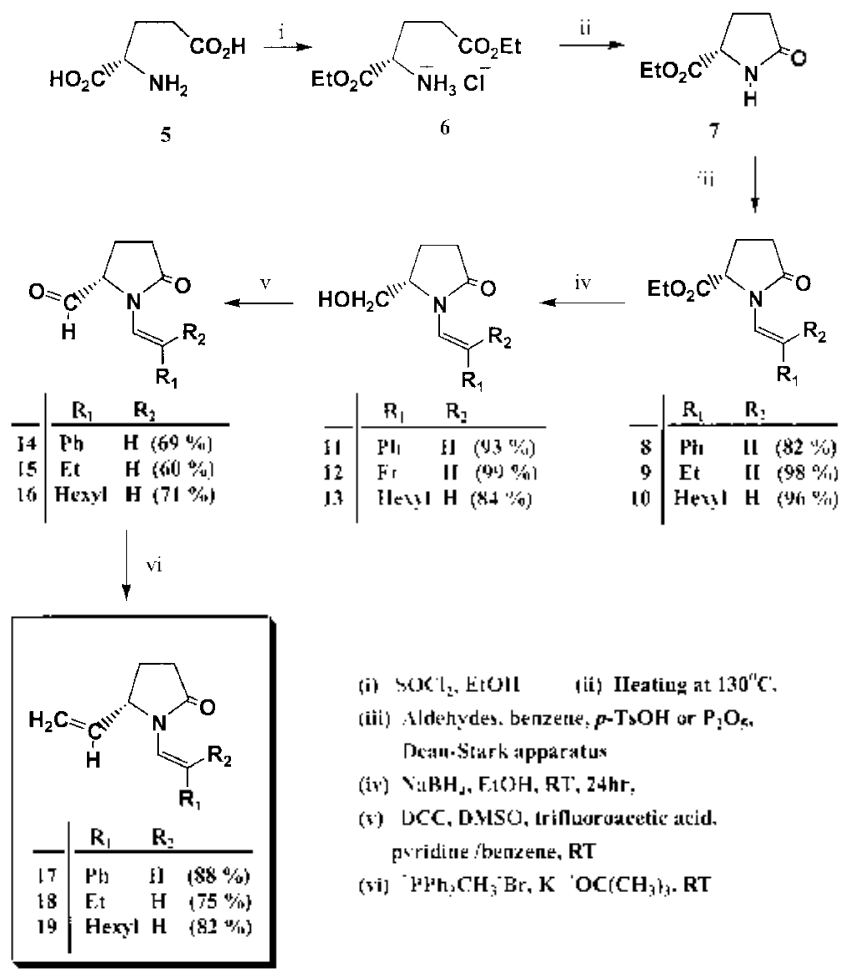

Scheme 1

yield by reacting L-glutamic acid with thionyl chloride in ethanol. ${ }^{10}$ Condensations of 7 with various aldehydes such as butanal, phenylacetaldehyde and octanal in the presence of $p$ - $\mathrm{TsOH}$ or $\mathrm{P}_{2} \mathrm{O}_{5}$ using Dean-Stark apparatus in benzene afforded the $N$-alkenyl lactams, 8-10." Subsequent reduction with sodium borohydride gave 11-13 in 84-99.8\% yield. The alkenyl substituents in the lactam ring appeared to be significant to prevent racemization during the next oxidation step. $N$-alkenyl aldelydes 14-16 were remarkably stable to neutral or basic condition but should be stored under argon atmosphere (below $0{ }^{\circ} \mathrm{C}$ ) to prevent the racemization. Stirring in a solution of DMSO, benzene, pyridine, trifluoroacetic acid and dicyclohexylcarbodimide (DCC) gave the corresponding aldehydes, 14-16 in 60-71\% yields. Wittig reactions of 14-16 with methyltriphenylphosphonium bromide in the presence of potassium t-butoxide for $10 \mathrm{hr}$ provided the $75-88 \%$ yields of $\mathbf{1 7 - 1 9}$. 
Table 1. The anticonvulsant evaluation of 17,18 and 19

\begin{tabular}{|c|c|c|c|c|c|c|c|c|c|}
\hline \multirow[t]{3}{*}{17} & Dose $(\mathbf{m g} / \mathbf{k g})$ & 75 & 100 & 125 & 150 & 175 & 200 & 225 & $\mathrm{ED}_{5(1}(\mathrm{mg} / \mathrm{kg})$ \\
\hline & $\mathrm{PTZ}^{u}$ & $4 / 4^{i}$ & $3 / 4$ & $3 / 4$ & $2 / 4$ & $1 / 4$ & - & $0 / 4$ & 153.1 \\
\hline & MES $^{h}$ & - & 44 & $3 / 4$ & $2 / 4$ & $1 / 4$ & - & - & 150.0 \\
\hline \multirow[t]{3}{*}{18} & Dose $(\mathbf{m g} / \mathbf{k g})$ & 25 & 50 & 75 & 100 & 150 & 200 & 225 & $\mathrm{ED}_{5(1)}(\mathrm{mg} / \mathrm{kg})$ \\
\hline & $\mathrm{PTZ}^{u}$ & $4 / 4^{2}$ & $3 / 4$ & $3 / 4$ & $3 / 4$ & $2 / 4$ & $1 / 4$ & $0 / 4$ & 134.4 \\
\hline & $\mathrm{MES}^{h}$ & $4 / 4^{2}$ & $3 / 4$ & $3 / 4$ & $2 / 4$ & $1 / 4$ & $0 / 4$ & - & 106.3 \\
\hline \multirow[t]{3}{*}{19} & Dose $(\mathbf{m g} / \mathbf{k g})$ & 75 & 100 & 125 & 150 & 175 & 200 & 225 & $\mathrm{ED}_{30}(\mathrm{mg} / \mathrm{kg})$ \\
\hline & $\mathrm{PTZ}^{u}$ & $4 / 4^{2}$ & 44 & - & $4 / 4$ & - & $4 / 4$ & 44 & Not Detected \\
\hline & $\mathrm{MES}^{b}$ & $4 / 4^{\circ}$ & 44 & - & $4 / 4$ & - & $4 / 4$ & 44 & Not Detected \\
\hline
\end{tabular}

"The subcutaneous pentylenetetrazole seizure threshold (PTZ) test entailed the administration of $80 \mathrm{mg} / \mathrm{kg}$ of pentylenetetrazole $\mathrm{n}$ the posterior midline of nice. This amount of pentylenetetrazole was expected to produce seizures in greater than $97 \%$ of nice. Maximal electroshock seizures (MES) were elicited with a 60 cycle alternating current of $50 \mathrm{~mA}$ intensity delivered for 0.2 second wia corneal electrodes. A drop of $0.9 \%$ saline was instilled in the eye prior to application of the electrodes so as to prevent the death of the animal. Protection in this test was defined as theabolition of the hind-limb tonic extension component of the seizure. "Convulsant aninal / treated animal

\section{Biological Anticonvulsant Activities}

It was reported that the MES test was correlated to the generalized tonic clonic seizure and the PTZ test to the generalized absence seizure. ${ }^{12}$ So these two kinds of seizure test were very meaningful for clinical prediction of the anticonvulsant drug candidates. Therefore, we investigated the anticonvulsant activity (phase 1 evaluation) with male ICR mice for compound 17, 18 and 19 in both the MES and PTZ test. Seizures were artificially induced by either electroshock and pentylenetetrazole and the animals were observed for 30 minutes.

As seen in Táble 1, compound $\mathbf{1 7}$ and $\mathbf{1 8}$ showed potential effects in both the MES and PTZ test. $N$-butenyl substituent compound 18 gave more stronger activities than phenyl (17) or octenyl (19) derivatives. In the MES test, $\mathrm{ED}_{50}$ value of 18 was $106.3 \mathrm{mg} / \mathrm{kg}$. It was found that $N$-substituents proved to be a major factor for the pharmacological activities. Phenyl analogue 17 showed less active anticonvulsant effect than 18 in both the MES and PTZ test and longer chain substituent such as 19 showed the lowest pharmacological activities.

In conclusion, we have described the synthesis of 5-vinylpyrrolidin-2-one derivatives containing the $N$-alkenyl moiety and compared their anticonvulsant activities. $N$-butenyl pyrrolidin-2-one derivatives 18 showed potent inhibitory activities in the MES and PTZ test. As $N$-substituents were longer and bigger, the anticonvulsant activities were decreased. Now we are currently continuing to prepare vinylGABA analogs to evaluate their anticonvulsant activities against picrotoxine and bicuculline convulsant in order to develop more active anticonvulsant prodrug candidate.

\section{Experimental Section}

Melting points were taken on a Haake Bucher melting point apparatus and are uncorrected. Infrared spectra were recorded with an infrared spectrophotometer model MagnaIR 500 and are reported in wave numbers. Both ${ }^{1} \mathrm{H}$ and ${ }^{15} \mathrm{C}$ NMR spectra were recorded on a FT-NMR Bruker 300 $(300 \mathrm{MHz})$ spectrometer and are reported in ppm using tetramethylsilane as the internal standard. $J$ values are given in $\mathrm{Hz}$. Mass spectra were recorded using a Shimazu QP 5000 spectrometer. High resolution mass spectra were obtained by electron impact at $70 \mathrm{eV}$ with a Kratos-MS- 50 instrument. Analytical thin-layer cluromatography was carried out on Merck 60 F254 silica gel plate and visualization was done with UV light, and/or by spraying with a $5 \%$ solution of phosphomolybdic acid followed by charring with a heat gun.

5-Hydroxymethyl-1-styryl-pyrrolidin-2-one, 11. To a solution of $1.12 \mathrm{~g}$ (29.61 mmol) of $\mathrm{NaBH}_{4}$ in $100 \mathrm{~mL}$. absolute ethanol was added $5.90 \mathrm{~g}(22.78 \mathrm{mmol})$ of 9 in 20 $\mathrm{mL}$ ethanol via syringe at $0{ }^{\circ} \mathrm{C}$. The solution was then stirred 75 hours at room temperature under argon atmosphere and the ethanol was evaporated under reduced pressure. The mixture was extracted with ether $\mathrm{H}_{2} \mathrm{O}$ (3 times) and dried over magnesium sulfate. Evaporation of solvent gave a colorless oil which was purified by silica gel column chromatograplyy ( $5 \%$ methanol $+95 \%$ methylene chloride, $\mathrm{Rf}=0.21)$ to give $5.46 \mathrm{~g}(21.29 \mathrm{mmol}, 93.5 \%)$ of 11 as a clear oil. $[\alpha]_{D}^{3 z}=-17.62^{\circ}(c=1.0$, methanol $) .{ }^{1} \mathrm{H}$ NMR $(300$ $\left.\mathrm{MHz}, \mathrm{CDCl}_{5}\right): \delta 7.55(1 \mathrm{H}, \mathrm{d},-\underline{\mathrm{HC}}=\mathrm{CPh}, J=15.18 \mathrm{~Hz})$, 7.46-7.02 $(5 \mathrm{H}, \mathrm{m}, \mathrm{Ph}), 6.01(1 \mathrm{H}, \mathrm{d},-\mathrm{C}=\mathrm{C} \underline{\mathrm{HPl}}, J=15.18$ $\mathrm{Hz}), 4.22-4.19(1 \mathrm{H}, \mathrm{q}), 4.03(1 \mathrm{H}, \mathrm{dd}, \mathrm{CHH}, J=11.43 \mathrm{~Hz})$. $3.75(1 \mathrm{H}, \mathrm{dd}, \mathrm{CH} \underline{\mathrm{H}}, J=11.43 \mathrm{~Hz}), 2.77-2.19(4 \mathrm{H}, \mathrm{m}$, lactam $\left.\mathrm{CH}_{2}\right)$ and $1.90-1.78(1 \mathrm{H}, \mathrm{s},-\mathrm{OH}, \mathrm{br}){ }^{13} \mathrm{C}$ NMR $(75 \mathrm{MHz}$. $\mathrm{CDCl}_{3}$ ): $\delta 174.92,136.63,129.13,127.13,125.99,122.85$, $112.68,61.95,58.45,31.21$ and 22.26 . Infrared (neat): $3393.1(\mathrm{br}), 3052.6,2937.8,1696.1,1684.5,1680.5(\mathrm{~s})$, $1645.5(\mathrm{~s}), 1597.6,1449.3(\mathrm{~s}), 1406.2(\mathrm{~s}), 1334.1,1286.8(\mathrm{~s})$, $1247.9,1070.7,1046.7,1013.2,950.9(\mathrm{~s}), 831.1,754.5(\mathrm{~s})$, 694.9(s) and, 649.1 $\mathrm{cm}^{-1}$. Mass Spectrum: CIMS m/s 218 $(\mathrm{M}+1)$.

1-But-1-enyl-5-hydroxylmethyl-pyrrolidin-2-one, 12. Reaction of $5.2 \mathrm{~g}(24.62 \mathrm{mmol}) 9$ with $2.33 \mathrm{~g}(61.55 \mathrm{mmol})$ of $\mathrm{NaBH}_{4}$ gave after silica gel column chromatography $(5 \%$ methanol $+95 \%$ methylene chloride, $\mathrm{Rf}=0.21) 4.16 \mathrm{~g}$ $(25.58 \mathrm{mmol}, 99.8 \%)$ of 12 as a yellow solid. $[\alpha]_{\mathrm{D}}^{32}=-2.2^{\circ}$ (c $\left.=0.056, \mathrm{CH}_{2} \mathrm{Cl}_{2}\right)$. ${ }^{1} \mathrm{H}$ NMR $\left(300 \mathrm{MHz}, \mathrm{CDCl}_{3}\right): \delta 6.73$ $(1 \mathrm{H}, \mathrm{d}, J=14.7 \mathrm{~Hz}), 5.12(1 \mathrm{H}, \mathrm{m}), 4.01-3.97(2 \mathrm{H}, \mathrm{m}), 3.61$ $(1 \mathrm{H}, \mathrm{m}), 3.11(1 \mathrm{H}, \mathrm{bs}, \mathrm{OH}), 2.18-2.04\left(4 \mathrm{H}\right.$, lactam $\left.\mathrm{CH}_{2}\right)$, 
2.62-2.38 $\left(2 \mathrm{H}, \mathrm{m}\right.$, vinyl $\left.\mathrm{CH}_{2}\right)$ and $0.99(3 \mathrm{H}, \mathrm{t}, J=7.40 \mathrm{~Hz})$. ${ }^{13} \mathrm{C} \mathrm{NMR}\left(75 \mathrm{MHz}, \mathrm{CDCl}_{3}\right.$ ): $\delta 174.2$ (lactam $\left.\mathrm{C}=0\right), 121.9$, $115.1,61.2,58.4,30.8,23.6,21.7$ and 14.5. Infrared (neat): $3400,1680,1410,1280$ and $1100 \mathrm{~cm}^{-1}$. Mass Spectrum: EIMS (m/s, relative intensity) $\mathrm{P}^{+} 169(2), 140\left(2,-\mathrm{CH}_{2} \mathrm{OH}\right)$, $126(100), 111,98,82,70$ and 55 .

1-Oct-1-enyl-5-hydroxylmethyl-pyrrolidin-2-one, 13. Reaction of $4 \mathrm{~g}(14.98 \mathrm{mmol}) 10$ with $0.85 \mathrm{~g}(22.48 \mathrm{mmol})$ $\mathrm{NaBH}_{4}$ gave after silica gel column chromatography $(5 \%$ methanol $+95 \%$ methylene chloride, $\mathrm{Rf}=0.27) 2.83 \mathrm{~g}$ $(12.58 \mathrm{mmol}, 84 \%)$ of 13 as a yellow oil. $[\alpha]_{\mathrm{V}}^{25}=-42.72^{\circ}(\mathrm{c}$ $=1.0$, methanol). ${ }^{1} \mathrm{H}$ NMR $\left(300 \mathrm{MHz}, \mathrm{CDCl}_{3}\right): \delta 6.75(1 \mathrm{H}$, d, - $\underline{\mathrm{HC}}=\mathrm{C}$-hexyl, $J=14.76 \mathrm{~Hz}), 5.01(1 \mathrm{H}$, quintet, $J=7.14$ $\mathrm{Hz}), 4.01-3.89\left(1 \mathrm{H}, \mathrm{m},-\mathrm{CHCH}_{2} \mathrm{OH}\right), 3.98-3.62(2 \mathrm{H}, \mathrm{m})$, 2.66-2.57 $(1 \mathrm{H}, \mathrm{m}), 2.40-2.30(1 \mathrm{H}, \mathrm{m}), 2.19-2.02(5 \mathrm{H}, \mathrm{m})$, $1.38-1.25\left(8 \mathrm{H}, \mathrm{m},\left(\mathrm{CH}_{2}\right)_{4}\right)$ and $0.87\left(3 \mathrm{H}, \mathrm{t},-\mathrm{CH}_{2} \mathrm{CH}_{2}, J=\right.$ $6.3 \mathrm{~Hz}) .{ }^{13} \mathrm{C}$ NMR $\left(75 \mathrm{MHz}, \mathrm{CDCl}_{3}\right): \delta 174.11,122.68$, $113.65,61.92,58.31,32.08,31.14,30.77,30.41,29.21$, 23.01, 22.09 and 14.49. Infrared (neat): 3397.9 (br, OH), $2955.2,2926.4,2855.0,2871.5,1680.1$ ( $\mathrm{s}, \mathrm{C}=\mathrm{O}$ ), 1655.1 (s, $\mathrm{NH}$ bend), 1457.9, 1413.4, 1343.7, 1282.7 (s, C-O), 1259.5, $1080.2,1056.3,1008.4,954.0,812.0,725.7$ and $653.9 \mathrm{~cm}^{-1}$. Mass Spectrum: CIMS m/s $226(\mathrm{M}+1)$.

5-Oxo-1-styryl-pyrrolidine-2-carbaldehyde, 14. Dissolution of $4.62 \mathrm{~g}$ ( $21.29 \mathrm{mmol}$ ) of 11 in $40 \mathrm{~mL}$ dry benzene was followed by addition of $52 \mathrm{~mL}$ dry DMSO. The clear solution was then treated with $2.32 \mathrm{~g}(2.37 \mathrm{~mL}, 29.33 \mathrm{mmol})$ of anhydrous pyridine (distilled from calcium hydride), 2.93 $\mathrm{g}(1.98 \mathrm{~mL}, 25.56 \mathrm{mmol})$ of trifluoroacetic acid, and $13.2 \mathrm{~g}$ (63.98 mmol) of dicyclohexylcarbodiimide in that order. The flask was tightly stoppered and stirred at room temperature for 18 hours under an argon atmosphere. Benzene (100 $\mathrm{mL}$ ) was added and the crystalline dicyclohexylurea was removed by filtration. The remaining solids were washed with benzene and the combined filtrates and washings were extracted with water (3 times) to remove DMSO. The organic layer was dried with magnesium sulfate, filtered and evaporated under reduced pressure. The crude product was purified by silica gel column chromatography ( $5 \%$ methanol $+95 \%$ methylene chloride, $\mathrm{Rf}=0.22)$ to give $3.17 \mathrm{~g}(14.74$ mmol, $69.3 \%)$ of 14 as a clear oil. $[\alpha]_{\mathrm{D}}^{\hat{2}}=-77.71^{\circ}(\mathrm{c}=1.0$, methanol). ' $\mathrm{H}$ NMR $\left(300 \mathrm{MHz}, \mathrm{CDCl}_{3}\right): \delta 9.65(1 \mathrm{H}, \mathrm{d}$, $\mathrm{CHO}, J=10.00 \mathrm{~Hz}), 7.66(1 \mathrm{H}, \mathrm{d}, \underline{\mathrm{HC}}=\mathrm{CPh}, J=15.06 \mathrm{~Hz})$, 7.34-7.17 $(5 \mathrm{H}, \mathrm{m}, \mathrm{Ph}), 5.80(1 \mathrm{H}, \mathrm{d}, \mathrm{HC}=\mathrm{CHPh}, J=15.09$ $\mathrm{Hz}), 4.50-4.45(1 \mathrm{H}, \mathrm{m}$, lactam $\mathrm{CH}), 2.63-2.57(2 \mathrm{H}, \mathrm{m}), 2.49$ $-2.38(1 \mathrm{H}, \mathrm{m})$ and $2.26-2.19(1 \mathrm{H}, \mathrm{m}) .{ }^{1} \mathrm{C}$ NMR $(75 \mathrm{MHz}$, $\left.\mathrm{CDCl}_{3}\right): \delta 199.70,174.10,135.89,129.16,127.54,126.21$, $123.18,113.21,64.22,30.15$ and 20.31. Infrared (neat): 3428.3, 3327.5(s), 3069.8, 3036.8, 2986.2, 2930.1(s), 2917.0, $2848.2(\mathrm{~s}), 2723.7,1952.1,1870.7,1739.5,1721.3,1685.5$ (m), 1648.1(s), 1598.3, 1492.2, 1450.6(s), 1431.3, 1375.1, (s), $1292.0(\mathrm{~s}), 1256.9$ (s), 1226.7(s), 1152.6(s), 1088.0, $1069.3,1021.6,957.3,947.5,840.6,824.2(s)$ and $757.4(s)$ $\mathrm{cm}^{-1}$. Mass Spectrum: CIMS m/s $216(\mathrm{M}+1)$.

1-But-1-enyl-5-oxo-pyrrolidine-2-carbaldehyde, 15. Reaction of $3 \mathrm{~g}$ ( $17.73 \mathrm{mmol})$ of 12 with $40 \mathrm{~mL}$ dry DMSO, $2.10 \mathrm{~g}(2.15 \mathrm{~mL}, 26.60 \mathrm{mmol})$ of pyridine, $3.05(2.06 \mathrm{~mL}$,
$26.60 \mathrm{mmol})$ of trifluoroacetic acid and $7.32 \mathrm{~g}$ ( $35.46 \mathrm{mmol})$ of dicyclohexylcarbodiimide gave after silica gel column chromatography ( $5 \%$ methanol $+95 \%$ methylene chloride, $\mathrm{Rf}=0.38) 1.77 \mathrm{~g}(10.59 \mathrm{mmol}, 60 \%)$ of $\mathbf{1 5}$ as a clear oil. ${ }^{1} \mathrm{H}$ $\operatorname{NMR}\left(300 \mathrm{MHz}, \mathrm{CDCl}_{3}\right): \delta 9.6(1 \mathrm{H}, \mathrm{d}, J=1.1 \mathrm{~Hz}), 6.9(1 \mathrm{H}$, $\mathrm{dd}, J=1.1,14.8 \mathrm{~Hz}), 5.1(1 \mathrm{H}, \mathrm{dt}, J=6.8,14.8 \mathrm{~Hz}), 4.3(1 \mathrm{H}$, $\mathrm{d}, J=2.3,13.7 \mathrm{~Hz}), 2.6-2.3(4 \mathrm{H}, \mathrm{m}), 2.1(2 \mathrm{H}, \mathrm{m})$ and 0.99 $(3 \mathrm{H}, \mathrm{t}, J=7.4 \mathrm{~Hz}),{ }^{13} \mathrm{C}$ NMR $\left(75 \mathrm{MHz}, \mathrm{CDCl}_{3}\right): \delta 200.0$, $173.0,122.4,115.3,64.0,29.9,23.4,19.9$ and 14.3. Infrared (neat): $3100,2300,1730,1680,1410,1070$ and $950 \mathrm{~cm}^{-1}$. Mass Spectrum: CIMS m/s $168(\mathrm{M}+1)$.

1-Oct-1-enyl-5-oxo-pyrrolidine-2-carbaldehyde, 16.

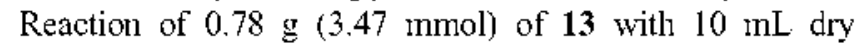
DMSO, $0.42 \mathrm{~g}(0.43 \mathrm{~mL}, 5.31 \mathrm{mmol})$ of pyridine, $0.60 \mathrm{~g}$ $(0.41 \mathrm{~mL}, 5.24 \mathrm{mmnol})$ of trifluoroacetic acid and $2.17 \mathrm{~g}$ (10.52 mmol) of dicyclohexylcarbdiimide gave after silica gel column chromatography $(5 \%$ methanol $+95 \%$ metlylene chloride, $\mathrm{Rf}=0.27) 0.55 \mathrm{~g}(2.47 \mathrm{mmol}, 71 \%)$ of 16 as a clear oil. $[\alpha]_{\mathrm{D}}^{35}=-67.97^{\circ}(\mathrm{c}=1.0$, methanol $){ }^{1} \mathrm{H}$ NMR $(300$ $\mathrm{MHz}, \mathrm{CDCl}): \delta 9.54(1 \mathrm{H}, \mathrm{d},-\mathrm{CHO}, J=3.36 \mathrm{~Hz}), 6.89(1 \mathrm{H}$, d, $\left.\underline{\mathrm{HC}}=\mathrm{CH}\left(\mathrm{CH}_{2}\right) \xi, J=14.67 \mathrm{~Hz}\right), 4.90-4.80(1 \mathrm{H}$, quintet, $\left.\mathrm{HC}=\mathrm{CH}-\left(\mathrm{CH}_{2}\right)_{3}\right), 4.31-4.25(1 \mathrm{H}, \mathrm{m}$, lactain $\mathrm{CH}), 2.58-2.30$ $\left(4 \mathrm{H}, \mathrm{m}\right.$, lactam $\left.\mathrm{CH}_{2}\right), 1.99-1.24\left(10 \mathrm{H}, \mathrm{m},-\left(\mathrm{CH}_{2}\right)^{-}\right)$and 0.86 $(3 \mathrm{H}, \mathrm{t}, J=6.93 \mathrm{~Hz}),{ }^{13} \mathrm{C}$ NMR $\left(75 \mathrm{MHz}, \mathrm{CDCl}_{2}\right): \delta 200.38$ (-CHO), 173.20 (lactan $\mathrm{C}=0$ ), 123.21, 114.31, 64.27, 32.00, $30.44,30.21,30.13,29.07,22.96,20.20$ and 14.46 . Infrared (neat): $3367.8(\mathrm{br}), 2955.2,2927.0(\mathrm{~s}), 2871.1,2855.0(\mathrm{~s}), 1702.6$, $1699.1,1679.4(\mathrm{~s}), 1661.8(\mathrm{~s}), 1559.3,1458.1,1411.2(\mathrm{~s})$, $1280.2(\mathrm{~s}), 1235.8,1113.8,1064.6(\mathrm{br}), 1013.2$ and $954.8(\mathrm{~s})$ $\mathrm{cm}^{-1}$. Mass Spectrum: CIMS m/s $224(\mathrm{M}+1)$.

1-Styryl-5-vinyl-pyrrolidin-2-one, 17. A stirred suspension of methyltriphenylphosphonium bromide $(3.90 \mathrm{~g}, 11.16$ mmol; dried in oven, oven temperature $150{ }^{\circ} \mathrm{C}$ for 30 minutes) in dry $25 \mathrm{~mL}$. THF (freshly distilled over calcium hydride) was treated with $1.33 \mathrm{~g}$ (11.90 $\mathrm{mmnol})$ of potassium tert-butoxide (high vacuuned prior to use) at room temperature. After the mixture was stirred for 30 minutes, $0.80 \mathrm{~g}$ ( $3.72 \mathrm{mmol}$ ) of $\mathbf{1 4}$ dissolved in dry $5 \mathrm{~mL}$ THF was then slowly added via syringe at room temperature and the whole reaction mixture was stirred for 15 hours at the same temperature. After this, the reaction mixture was quenched with water and extracted with ether ( 3 times). The combined extracts were dried with magnesium sulfate, filtered, evaporated and purified by silica gel column chromatography (methylene chloride, $\mathrm{Rf}=0.3)$ to give $0.69 \mathrm{~g}(3.27 \mathrm{mmol}, 88$ $\%)$ of 17 as a pale yellow oil. $[\alpha]_{\mathrm{D}}^{25}=-11.72^{\circ}(\mathrm{c}=1.0$, methanol). ${ }^{1} \mathrm{H}$ NMR $\left(300 \mathrm{MHz}, \mathrm{CDCl}_{3}\right): \delta 7.53(1 \mathrm{H}, \mathrm{d}$, $-\underline{\mathrm{HC}}=\mathrm{CHPl}, J=15.01 \mathrm{~Hz}), 7.31-7.11(5 \mathrm{H}, \mathrm{m},-\mathrm{Ph}), 5.92(1 \mathrm{H}$, $\mathrm{d},-\mathrm{CH}=\mathrm{C} \underline{\mathrm{HPh}}, J=15.01 \mathrm{~Hz}), 5.84-5.75\left(1 \mathrm{H}, \mathrm{m},-\mathrm{C} \underline{\mathrm{H}}=\mathrm{CH}_{2}\right)$, 5.23-5.16 $\left(2 \mathrm{H}, \mathrm{m},-\mathrm{CH}=\mathrm{CH}_{2}\right), 4.51-4.47(1 \mathrm{H}, \mathrm{m}$, lactam $\mathrm{CH}), 2.58-1.77(4 \mathrm{H}, \mathrm{m}),{ }^{13} \mathrm{C}$ NMR $\left(75 \mathrm{MHz}, \mathrm{CDCl}_{3}\right): \delta$ $174.06,136.84,136.51,129.05,127.00,126.02,123.01$, $115.81,113.66,59.67,29.30$ and 26.14. HRMS calculated for $\mathrm{C}_{14} \mathrm{H}_{15} \mathrm{NO} 213.115124$; Found 213.115364 .

1-But-1-enyl-5-vinyl-pyrrolidin-2-one, 18. Reaction of $0.81 \mathrm{~g}(4.86 \mathrm{mmol})$ of $15,5.2 \mathrm{~g}(14.6 \mathrm{mmol})$ of methyltriphenylphosphonium bromide and $1.7 \mathrm{~g}(15.2 \mathrm{mmol})$ of 
potassium tert-butoxide gave after silica gel column chromatography (methylene chloride, $\mathrm{Rf}=0.30) 0.61 \mathrm{~g}(3.65 \mathrm{mmol}$, $75 \%)$ of 18 as a clear oil. $[\alpha]_{\mathrm{D}}^{25}=-45.8^{\circ}(\mathrm{c}=0.035$, $\left.\mathrm{CH}_{2} \mathrm{Cl}_{2}\right),{ }^{l} \mathrm{H}$ NMR $\left(300 \mathrm{MHz}, \mathrm{CDCl}_{3}\right): \delta 6.75(1 \mathrm{H}, \mathrm{d}, J=$ $14.5 \mathrm{~Hz}), 5.70(2 \mathrm{H}, \mathrm{m}), 5.19(1 \mathrm{H}, \mathrm{m}), 5.10(1 \mathrm{H}, \mathrm{m}), 4.35$ $(1 \mathrm{H}, \mathrm{t}, J=7.4 \mathrm{~Hz}), 2.6-1.8(6 \mathrm{H}, \mathrm{m})$ and $0.99(3 \mathrm{H}, \mathrm{t}, J=7.4$ $\mathrm{Hz}) .{ }^{13} \mathrm{C} \mathrm{NMR}\left(75 \mathrm{MHz}, \mathrm{CDCl}_{3}\right): \delta 174.1,136.9,122.0$, $116.0,115.7,59.4,29.7,25.8,23.5$ and 14.4. HRMS calculated for $\mathrm{C}_{10} \mathrm{H}_{1}$ NO 165.11538; Found 165.11622.

1-Oct-1-enyl-5-vinyl-pyrrolidin-2-one, 19. Reaction of $0.55 \mathrm{~g}(2.47 \mathrm{mmol})$ of $16,2.64 \mathrm{~g}$ (7.40 mmole) of methyltriphenylphosphonium bromide and $1.38 \mathrm{~g}(12.35 \mathrm{mmol})$ of potassium tert-butoxide gave after silica gel column chromatography (methylene chloride, $\mathrm{Rf}=0.30) 0.449 \mathrm{~g}(2.03 \mathrm{mmol}$, $82 \%)$ of 19 as a clear oil. $[\alpha]_{\mathrm{D}}^{\frac{15}{5}}=-20.17^{\circ}(\mathrm{c}=1.0$, methanol). 'H NMR (300 MHz, CDCl $): \delta 6.77(1 \mathrm{H}$, d, $-\underline{H C}=\mathrm{CHR}, J=14.6 \mathrm{~Hz}), 5.81-5.69(1 \mathrm{H}, \mathrm{m},-\mathrm{CH}=\mathrm{C} \underline{\mathrm{HR}})$, $5.19-4.96\left(3 \mathrm{H}, \mathrm{m}, \mathrm{CH}_{2}=\mathrm{CH}-\right), 4.34(1 \mathrm{H}, \mathrm{m},-\mathrm{C} \underline{\mathrm{H}}-\mathrm{N}), 2.57-$ $2.23\left(4 \mathrm{H}, \mathrm{m}\right.$, lactam $\left.\mathrm{CH}_{2}\right), 2.05-1.98\left(2 \mathrm{H}, \mathrm{m},-\mathrm{C}=\mathrm{CH}-\mathrm{CH}_{2}\right)$, $1.26(8 \mathrm{H}, \mathrm{m})$, and $0.87\left(3 \mathrm{H}, \mathrm{t},-\mathrm{CH}_{\mathrm{i}}\right) .{ }^{17} \mathrm{C} \mathrm{NMR}(75 \mathrm{MHz}$, $\left.\mathrm{CDCl}_{3}\right): \delta 174.05,136.98,122.78,116.38,115.73,59.75$, $32.04,30.66,30.34,30.05,29.06,26.10,22.97$ and 14.44 Infrared (neat): $3473.7(\mathrm{br}), 2955.9,2925.8(\mathrm{~s}), 2871.6,2854.8$ (s) $1707.8(\mathrm{~s}), 1664.2(\mathrm{~s}), 1458.7,1398.3(\mathrm{~s}), 1338.9,1287.1$ (m), 1234.3, 994.0 and $951.8(\mathrm{~s}) \mathrm{cm}^{-1}$. Mass Spectrum: CIMS m/s $222(\mathrm{M}+1)$. HRMS calculated for $\mathrm{C}_{14} \mathrm{H}_{23} \mathrm{NO}$ 221.17785; Found 221.17736

Acknowledgment. This work was supported by grants from the 98 Good Health R \& D Project, Ministry of Health and Welfare, R. O. K and the Kyungsung University Research Grants in 2002 (2002-3029).

\section{References}

1. (a) Metcalf, B. W. Biochemical Phamacology 1979, 28,1705 and references cited therein. (b) Ommaya. A. K. Adr: Nemologr 1976 . 15,332 .

2. Barbeau. A. Lancet II 1973. 1499.

3. Roberts, E. Biochem. Pharmac. 1974, $23,2637$.

4. Pery, T. L.; Hansen, S.; Kloster, M. N. New Engl J. Med. 1973, $288,337$.

5. Palfreymen, M. G.; Heot, S.; Lippert, B.; Schechter. P. J. Etti $d$. Phaimas. 1978. 50.325.

6. (a) Roberts. E.; Kuriyama. K. Brain Res. 1968. 8. 1. (b) Roberts. E. Neurosci. Res. Program Bull. 1972, If, 468.

7. (a) Silverman, R. B. Methods Enzmol. 1995, 249, 240. (b) Tassinari, C. A.; Michelucei, R.: Ambrosetto, G.; Salvi, F. Arch. Netmol, 1987, 44, 907. (c) Browne. T. R.; Mattson. R. J.; Penry, J. K.; Smith. D. B.; Treiman, D. M.; Wilder, B. J.; Ben-Mensachem. E; Miketta. R. M.; Sheryy. K. M.; Szabo. G. K. Br. J. Clm. Phamaco. 1989, 27, 952. (d) Silvemann, R. B.: Levy, M. A. Biochem. Biophys. Res. Commam. 1980, 95, 250. (e) Silvermann, R. B.; Levy, M. A. Biochemistry 1981, 201 1197. (f) Silvermann, R. B.; Levy, M. A.; Muztar, A. J.; Hirsch. J. D. Biochem. Biophrs. Res. Conmum. 1981, 102. 520. (g) Silvermann. R. B.; Muztar, A. J.; Levy. M. A.; Hirsch. J. D. Life Sci. 1983, 32, 2717. (h) Silvermann, R. B. Mechanism-Based Enzyme Inactivation: Chemistry and Enymologr'; CRC Press: Boca Raton, 1988; Vols. I and II. (i) Silvemann, R. B.; Inverdo, B. J. Biochemistry 1986, 25. 6817 .

8. (a) Zezza. C. A.; Kwon. T, W.; Shell. J. ; Smith. M. B. Heterockcles 1992, 34. 1325. (b) Smith, M. B.; Shroff. H. N. J. Org. Chen. $1984,49,2900$.

9. Kwon, T. W.; Keusenkothen, P. F.; Smith, M. B. J. Org. Chem. $1992,57,6169$.

10. (a) Silvemnann. R. B.; Levy. M. A. J. Org. Chem. 1980, 45 815 (b) Adkins. H.; Billica, H. R. J. An. Chem. Soc. 1948, 70 . 3121 .

11. (a) Zezza, C. A.; Smith, M. B. Sinth Commun. 1987, 17, 729. (b) Smith, M. B.; Shroff, H. N. Heterocycles 1985, 23 2229 .

12. (a) Swinyard, E. A.: Woodhead, J. H.; White, H. S.; Franklin, M. R. General Principles, Experimental Section, Otantification and El'altation of Anticon'ulsants in Antiepileptic Drtigs, $3^{\text {rd }}$ Ed.; Levy. R. Ed.; Ravan Press: New York 1988; p 88. (b) Krall. R. L.: Penry, J. K.; White, B. G.; Kupferberg, H. J.; Swinyard, C. A. Epilepsia 1978, 19, 409. 